01

\title{
Методика измерения сечений многофотонного поглощения лазерных импульсов в газах с помощью спектрофона
}

\author{
(C) А.Н. Куряк, Б.А. Тихомиров \\ Институт оптики атмосферы им. В.Е. Зуева Сибирского отделения РАН, \\ 634055 Томск, Россия \\ e-mail: kurjak@iao.ru
}

Поступила в редакцию 12.04.2021 г.

В окончательной редакции 29.04.2021 г.

Принята к публикации 04.05.2021 г.

Разработана методика измерения сечений многофотонного поглощения гауссовых лазерных импульсов в газах с помощью оптико-акустического детектора с временным разрешением сигналов (спектрофона). Работоспособность методики протестирована на примере однофотонного и трехфотонного поглощения наносекундных импульсов квазимонохроматичного излучения с длиной волны $266 \mathrm{~nm}$ молекулярным кислородом.

Ключевые слова: многофотонное поглощение, кислород, гауссов лазерный импульс, оптико-акустический детектор (спектрофон).

DOI: 10.21883/OS.2021.08.51194.2154-21

\section{Введение}

В последние годы значительно повысился интерес к изучению распространения высокоинтенсивных лазерных импульсов в атмосферном воздухе. Интерес связан прежде всего с эффектами самофокусировки и филаментации лазерных пучков [1-5], генерации гармоник [6] и суперконтинуума $[7,8]$ и с практической реализацией дистанционных методов измерения параметров атмосферы с использованием фемтосекундных лазеров [9]. Еще до достижения филаментации лазерных пучков излучение в значительной степени ослабляется из-за многофотонного поглощения основными молекулярными составляющими атмосферного воздуха - азотом, кислородом и водяным паром $[10,11]$, что наиболее заметно проявляется для излучения УФ диапазона спектра $[12,13]$. В связи с этим количественные данные о характеристиках многофотонного поглощения лазерного излучения атмосферным воздухом и его основными молекулярными составляющими представляют большой интерес.

Для характеристики линейного (однофотонного) поглощения в спектроскопии атмосферных газов применяется интенсивность линии поглощения (с размерностью $\left.\left[\mathrm{cm} \mathrm{mol}^{-1}\right]\right)$, которая соответствует площади под кривой контура линии поглощения, приведенной к одной молекуле поглощающего газа [14]. Интенсивности линий относятся к конкретным колебательно-вращательным (КВ) переходам молекул атмосферных газов в основном электронном состоянии и наряду с коэффициентами уширения и сдвига линий давлением воздуха систематизированы в базе данных HITRAN [15]. Эти данные широко используются при решении задач оптики атмосферы - расчетах радиационного баланса Земли, спут- никовых и лидарных измерениях содержания газовых составляющих атмосферы на разных высотах [16].

Многофотонное поглощение наблюдается при поглощении мощного излучения импульсных лазеров, и оно зависит не только от спектроскопических свойств газа, но и от интенсивности излучения. Поэтому для характеристики n-фотонного поглощения высокоинтенсивных лазерных импульсов применяется сечение поглощения $\sigma^{(n)}$ [17] с размерностью $\left[\mathrm{cm}^{2 n} \mathrm{~s}^{n-1}\right]$, которое в законе Бугера-Ламберта-Бэра для линейного поглощения соответствует коэффициенту поглощения квазимонохроматичного излучения с фиксированной длиной волны, приведенному к одной молекуле поглощающего газа [14]. Эксперименты, направленные на исследования многофотонного поглощения лазерных импульсов в газах, обычно проводятся со сфокусированными лазерными пучками. Для этих целей лучше всего подходят оптико-акустический ОА [18] и оптико-гальванический (ОГ) [19] методы лазерной спектроскопии.

В отличие от ОГ метода, при реализации которого измеряется плотность свободных электронов в канале распространения лазерного импульса, ОА методом измеряется амплитуда сигнала давления, генерируемого в результате поглощения излучения и последующего увеличения поступательной энергии атомов и молекул из-за столкновений друг с другом. Если ОГ сигнал генерируется в результате ионизации, то ОА сигнал может генерироваться в результате целого ряда физических механизмов: поглощения на КВ и электронных переходах молекул, ВКР, ионизации и диссоциации, поглощения излучения продуктами фотохимических реакций и др. Оптико-акустический метод отлично зарекомендовал себя в линейной лазерной спектроскопии в видимом, ИК и микроволновом диапазонах спектра и в газовом анализе. 


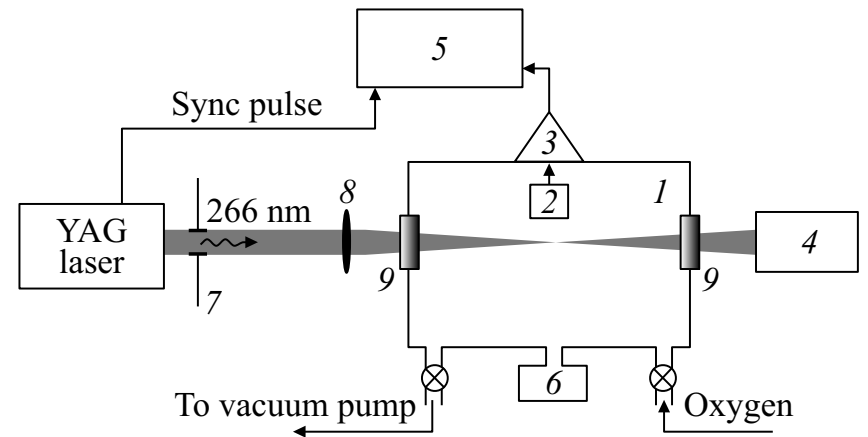

Рис. 1. Схема установки. $1-$ ОАД, $2-$ микрофон, $3-$ усилитель, 4 - калориметр, 5 - осциллограф, 6 - вакуумметр, 7 - диафрагма, 8 - линза, 9 - оптические окна.

В УФ области работоспособность ОА метода, на наш взгляд, изучена недостаточно.

В настоящей работе мы представляем разработанную методику ОА измерений сечений многофотонного поглощения лазерных импульсов с гауссовым распределением интенсивности излучения. Методика тестируется на примере одно- и трехфотонного поглощения квазимонохроматичного излучения четвертой гармоники YAG-лазера однокомпонентным газом - кислородом. При этом для калибровки ОА детектора (ОАД, или спектрофона [18]) используются литературные данные о сечении линейного поглощения, а сечение трехфотонного поглощения излучения $266 \mathrm{~nm}$ кислородом является искомой величиной.

Результаты работы представляют интерес для специалистов в области лазерной спектроскопии атмосферных газов и взаимодействия мощного лазерного излучения с веществом.

\section{Экспериментальная установка}

Схема экспериментальной установки представлена на рис. 1. Лазер (модель LS-2134U, Минск) с нелинейными преобразователями частоты основного излучения в гармоники позволяет получать импульсы линейно поляризованного излучения на длине волны $\lambda=266 \mathrm{~nm}$ длительностью $\tau_{0.5}=6 \mathrm{~ns}$ (FWHM) с частотой повторения до $15 \mathrm{~Hz}$. Радиус пучка за диафрагмой 7 составляет $5 \mathrm{~mm}$. Энергия лазерных импульсов варьируется путем дискретного изменения напряжения на лампевспышке и измеряется с помощью серийного калориметpa Ophir с измерительной головкой 12A.

Оптико-акустический детектор 1 включает в себя цилиндрическую камеру диаметром $20 \mathrm{~cm}$ и длиной $25 \mathrm{~cm}$. Для ввода и вывода излучения на торцах камеры вмонтированы оптические окна 9 из кварца КУ. Внутри камеры в фокальной плоскости линзы 8 с фокусным расстоянием $F=100 \mathrm{~cm}$ установлен конденсаторный микрофон МК-3012. При поглощении части энергии лазерного импульса в камере ОАД генерируется акустический биполярный импульс [20], который с помощью микрофона преобразуется в электрический сигнал. Электрический сигнал микрофона усиливается широкополосным усилителем 3 и регистрируется цифровым осциллографом TDS 1012 5. Необходимо отметить, что для ОАД с временным разрешением сигналов в отсутствие поглощения в исследуемом газе амплитуда ОА сигнала $U_{0}=0$. В настоящем эксперименте регистрировались зависимости амплитуды $U_{0}$ (pk-pk) от энергии лазерных импульсов $E$ с синхронным накоплением 64 сигналов ОАД для каждого значения энергии лазерного импульса.

Перед измерениями камера ОАД несколько раз откачивалась до остаточного давления $P \sim 0.04 \mathrm{mbar}$ и промывалась кислородом. После чего в камеру напускался кислород $(99.999 \%)$ производства ООО „ПГСсервис“". Давление газа в камере ОАД контролировалось с помощью вакуумметров DVR-5 и ВИT-2 6.

\section{Генерация ОА сигнала при многофотонном поглощении гауссовых лазерных импульсов}

Амплитуда сигнала $U_{0}$ при многофотонном поглощении лазерных импульсов с гауссовым распределением интенсивности излучения однокомпонентным газом состоит из вкладов составляющих $U_{0}^{(n)}[21]$ :

$$
\begin{aligned}
& U_{0}=\sum_{1}^{n} U_{0}^{(n)} \approx \sum_{1}^{n} \alpha n^{3 / 4}\left(\sigma^{(n)} N n^{-3 / 2} I_{0}^{n-1}\right) E \\
& =\sum_{1}^{n} \alpha \sigma^{(n)} N n^{-3 / 4}\left(\frac{2 \sqrt{\ln 2}}{\pi^{3 / 2} r_{0}^{2} \tau_{0,5}}\right)^{n-1} E^{n}
\end{aligned}
$$

где $\alpha-$ чувствительность ОАД при линейном (однофотонном) поглощения, $\sigma^{(n)}-$ сечение $n$-фотонного поглощения. Число молекул поглощающего газа $N=L P$, $L=2.68 \cdot 10^{19} \mathrm{~cm}^{-3}$ - число Лошмидта, $P$ - давление поглощающего газа, $n=1,2,3, \ldots \mathrm{B}(1)$ сомножитель $\left(\sigma^{(n)} N n^{-3 / 2} I_{0}^{n-1}\right)$ имеет смысл эффективного коэффициента $n$-фотонного поглощения, выраженного через пиковую интенсивность гауссова импульса:

$$
I_{0}=\frac{2 E}{\pi^{3 / 2} r_{0}^{2} \tau},
$$

где $\tau=\tau_{0,5} / \sqrt{\ln 2}, r_{0}-$ радиус пучка, при котором интенсивность $I\left(r_{0}\right)=I_{0} e^{-2}$.

Для сечения $n$-фотонного поглощения из выражения (1) легко получить:

$$
\sigma^{(n)} \approx b_{n} n^{3 / 4}\left[\alpha\left(\frac{2 \sqrt{\ln 2}}{\pi^{3 / 2} r_{0}^{2} \tau_{0,5}}\right)^{n-1} L P E^{n-1}\right]^{-1},
$$

где $b_{n}$ - коэффициент подгонки соответствующей составляющей $U_{0}^{n}$ в экспериментальной зависимости $U_{0}(E)$ 


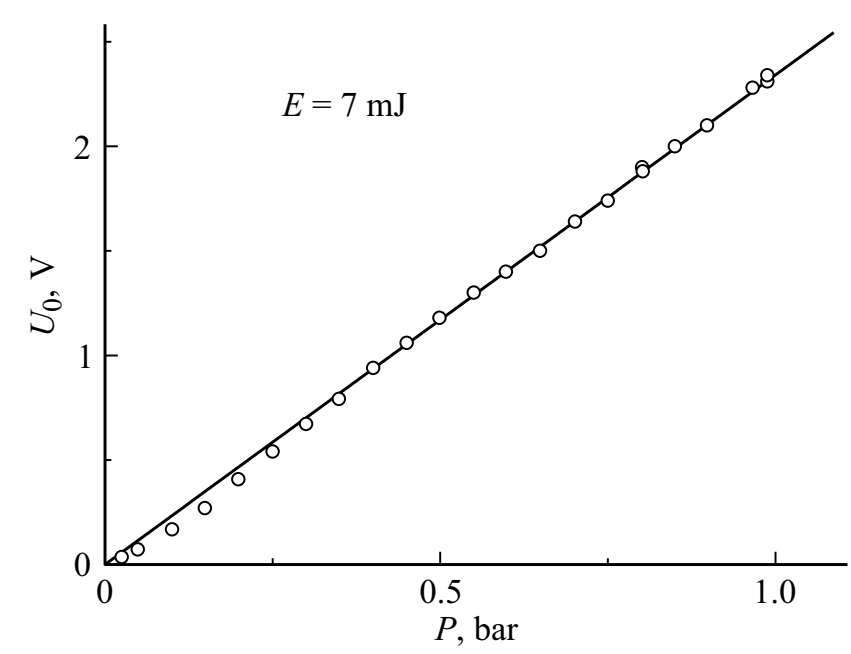

Рис. 2. Амплитуда ОА сигнала в зависимости от давления кислорода.

полиномом $n$-й степени. Физические величины имеют следующие размерности: $\sigma^{(n)}\left[\mathrm{cm}^{2 n} \mathrm{~s}^{n-1} \mathrm{~J}^{-n}\right], \alpha\left[\mathrm{V} \mathrm{J}^{-1} \mathrm{~cm}\right]$, $P[\mathrm{bar}], E[\mathrm{~J}]$. Чтобы в формуле (2) перейти к классической размерности сечения многофотонного поглощения [17], необходимо значения, полученные по формуле $(2)$, делить $(n-1)$ раз на число $1.33 \cdot 10^{18}-$ количество фотонов в лазерном импульсе с энергией $1 \mathrm{~J}$.

Таким образом, для того чтобы определить сечение $n$ фотонного поглощения в однокомпонентном газе, необходимо аппроксимировать экспериментальные зависимости $U_{0}(E)$ полиномом $n$-й степени и по найденным коэффициентам подгонки $b_{n}$ с помощью (2) вычислить значения сечения поглощения. Для увеличения вклада в зависимость $U_{0}(E)$ от линейного поглощения необходимо проводить измерения с коллимированными лазерными пучками, или с применением длиннофокусных линз. Чтобы увеличить вклад в $U_{0}(E)$ от многофотонного поглощения, необходимо применять короткофокусные линзы.

\section{Результаты и их обсуждение}

На рис. 2 представлена зависимость амплитуды $U_{0}$ от давления кислорода. Из рисунка видно, что во всей области изменения давления кислорода амплитуда ОА сигнала растет прямо пропорционально давлению. Небольшие отклонения экспериментальных точек от линейной зависимости, которые наблюдаются в области давлений $P \leq 0.25$ bar, можно объяснить увеличением времени дезактивации возбужденных молекул с уменьшением давления [18]. То есть чувствительность ОАД в области $P \leq 0.25$ bar зависит нелинейно от давления кислорода. Поэтому дальнейшие измерения зависимостей $U_{0}(E)$ проводились при давлениях кислорода $P \geq 0.25$ bar.

На рис. 3 представлены зависимости амплитуды ОА сигнала от энергии лазерных импульсов с длиной вол- ны излучения $266 \mathrm{~nm}$ при четырех значениях давления кислорода $P=0.25,0.5,0.75$ и 1 bar. Из рисунка видно, что амплитуда $U_{0}$ растет пропорционально давлению. В области изменения энергии лазерных импульсов от 0.7 до $8 \mathrm{~mJ}$ (пиковой интенсивности излучения $I_{0}$ от $0.1 \mathrm{GW} / \mathrm{cm}^{2}$ до $1.1 \mathrm{GW} / \mathrm{cm}^{2}$ ) степень многофотонности $n$ изменяется. Из литературы известно, что поглощение излучения с длиной волны около $266 \mathrm{~nm}$ кислородом определяется слабым линейным поглощением в электронной полосе Герцберга I [22] и трехфотонной ионизацией молекул $\mathrm{O}_{2}[13,23]$. Первый потенциал ионизации для молекул кислорода составляет величину $U_{i}=12.1 \mathrm{eV}$, и энергии фотона $h v=4.7 \mathrm{eV}$ излучения с длиной волны $266 \mathrm{~nm}$ вполне достаточно для трехфотонной ионизации молекул $\mathrm{O}_{2}$.

Сплошными и штриховыми линиями на рис. 3 представлены результаты аппроксимации экспериментальных зависимостей полиномом третей степени $U_{0}=b_{1} E+b_{2} E^{2}+b_{3} E^{3}$. При подгонке в соответствии с вышеизложенным коэффициент $b_{2}$ принимался равным нулю. Сплошные линии - результат подгонки с учетом относительной погрешности измерений амплитуды ОА сигнала $\delta U_{0} / U_{0}=0.05$. Штриховые линии - результат подгонки без учета погрешностей измерения $U_{0}$. При построении рисунка учтены потери энергии лазерных импульсов в 10\% на выходном окне ОАД.

Численные значения коэффициентов аппроксимации $b_{1}$ и $b_{3}$ с учетом погрешностей измерений $U_{0}$ для четырех значений давления кислорода представлены в табл. 1.

Анализ данных в таблице показывает, что коэффициенты $b_{1}$ и $b_{3}$ коррелируют с давлением кислорода. Из рассмотрения рис. 3 видно, что учет погрешностей приводит к более хорошему совпадению экспериментальных точек со сплошными подгоночными кривыми. Если не учитывать погрешности, то штриховые подгоночные кривые в области малых энергий отклоняют-

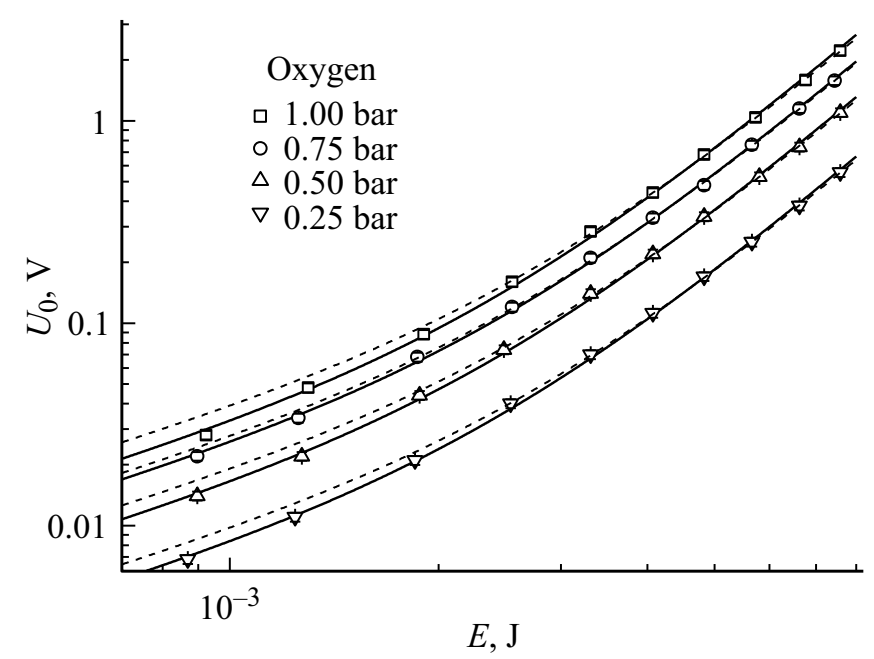

Рис. 3. Амплитуда ОА сигнала в зависимости от энергии лазерных импульсов $266 \mathrm{~nm}$. 
Таблица 1. Коэффициенты аппроксимации экспериментальных данных

\begin{tabular}{c|c|c}
\hline$P$, bar & $b_{1}$ & $10^{-6} b_{3}$ \\
\hline 1 & $28.3(1.1)$ & $4.75(0.13)$ \\
0.75 & $22.5(1.1)$ & $3.49(0,13)$ \\
0.5 & $14.2(1.1)$ & $2.34(0.13)$ \\
0.25 & $7.1(1.1)$ & $1.19(0.13)$
\end{tabular}

Примечание. В скобках приведены СКО подгонки.

Таблица 2. Сечения поглощения $\sigma^{(1)}$ и $\sigma^{(3)}$ излучения лазерных импульсов 266 нм кислородом

\begin{tabular}{c|c|c}
\hline$P$, bar & $\sigma^{(1)},\left(10^{-25} \mathrm{~cm}^{2}\right)$ & $\sigma^{(3)},\left(10^{-77} \mathrm{~cm}^{6} \mathrm{c}^{2}\right)$ \\
\hline 1 & $9.4(0.4)$ & $1.34(0.04)$ \\
0.75 & $10.0(0.6)$ & $1.32(0.05)$ \\
0.5 & $9.5(0.7)$ & $1.32(0.07)$ \\
0.25 & $9.6(1.5)$ & $1.35(0.15)$
\end{tabular}

Примечание. В скобках приведены погрешности, соответствующие СКО подгоночных коэффициентов в табл. 1.

ся от экспериментальных зависимостей, что связано с уменьшением веса малых значений $U_{0}$ слева на рисунке в подгонке методом наименьших квадратов.

В соответствии с формулой (1) при линейном поглощении амплитуда $U_{0}$ определяется простым соотношением $U_{0}=\alpha \sigma^{(1)} L P E=b_{1} E$. Для кислорода сечение поглощения $\sigma^{(1)}=9.44 \cdot 10^{-25} \mathrm{~cm}^{2} \quad[23]$. Для калибровки ОАД используем значение коэффициента $b_{1}$ из табл. 1 при $P=1$ bar, поскольку при этом давлении отношение сигнал/шум для составляющей линейного поглощения в сигнале ОАД максимально. Таким образом, чувствительность ОАД по линейному поглощению составляет величину $\alpha=28.3 /\left(9.44 \cdot 10^{-25} \cdot 2.68 \cdot 10^{19}\right)=1.12 \cdot 10^{6} \mathrm{~V} \cdot \mathrm{cm} \cdot \mathrm{J}^{-1}$. При $E=1 \mathrm{~J}, r_{0}=\sqrt{\frac{\lambda F}{2 \pi}}=2.06 \cdot 10^{-2} \mathrm{~cm} \mathrm{[24]} \mathrm{и} \tau_{05}=6 \mathrm{~ns}$ пиковая интенсивность $I_{0}=141 \mathrm{GW} / \mathrm{cm}^{2}$.

После подстановки всех численных значений в (2) получаем простые соотношения: $\sigma^{(1)} \approx 3.33 \cdot 10^{-26} b_{1} / P$ и $\sigma^{(3)} \approx 2.83 \cdot 10^{-84} b_{3} / P$, которые используем для вычисления сечений поглощения (табл. 2).

Из таблицы видно, что восстановленные сечения поглощения $\sigma^{(1)}$ равны или близки к литературному значению [23]. Наибольшее отклонение $\sigma^{(1)}$ от литературного значения наблюдается для зависимости $U_{0}(E)$ при $P=0.75 \mathrm{bar}$, однако и оно не выходит за рамки совместной погрешности восстановления $\sigma^{(1)}$ при двух разных давлениях кислорода. Восстановленные значения $\sigma^{(3)}$ из всех четырех зависимостей $U_{0}(E)$ на рис. 3 совпадают с точностью до второго знака после запятой, что говорит о высоком отношении сигнал/шум в измерениях $U_{0}$ при больших энергиях лазерного импульса и работоспособности созданной методики. Учет погрешности измерений амплитуды ОА сигнала $U_{0}$ в аппроксимации экспериментальных зависимостей дает значения $\sigma^{(3)}$, которые на $20 \%$ превышают значения $\sigma^{(3)}$, полученные без учета погрешностей. Найденное в настоящей работе значение $\sigma^{(3)}=(1.3 \pm 0.3) \cdot 10^{-77} \mathrm{~cm}^{6} \mathrm{~s}^{2}$ в 2.2 раза больше значения $\sigma^{(3)}=(0.6 \pm 0.2) \cdot 10^{-77} \mathrm{~cm}^{6} \mathrm{~s}^{2}$ из работы [25]. Различие можно объяснить тем, что в [25] не было учтено ослабление излучения фокусирующей линзой и входным окном спектрофона, а обработка экспериментальных зависимостей производилась без учета погрешностей измерения $U_{0}$.

\section{Заключение}

В настоящей работе создана методика измерения сечений многофотонного поглощения в газах с помощью спектрофона. Основой для методики служит полученное математическое выражение для амплитуды ОА сигнала, генерируемого при многофотонном поглощении лазерных импульсов с гауссовым распределением интенсивности. Методика заключается в регистрации в эксперименте зависимости амплитуды ОА сигнала от энергии лазерного импульса, аппроксимации этой зависимости полиномом и оценки вкладов в амплитуду ОА сигнала от поглощения с разной степенью многофотонности.

Работоспособность методики протестирована на примере однофотонного и трехфотонного поглощения сфокусированного квазимонохроматичного излучения лазерных импульсов с длиной волны излучения $266 \mathrm{~nm}$ молекулярным кислородом при давлениях $P=0.25,0.5,0.75$ и 1 bar. Калибровка спектрофона производилась по известному однофотонному поглощению лазерных импульсов $266 \mathrm{~nm}$ в кислороде. Обработка экспериментальных зависимостей $U_{0}(E)$, зарегистрированных для четырех значений давления кислорода, дала одинаковые значения сечения трехфотонного поглощения молекулярным кислородом $\sigma^{(3)}=(1.3 \pm 0.3) \cdot 10^{-77} \mathrm{~cm}^{6} \mathrm{~s}^{2}$.

\section{Финансирование работы}

Работа выполнена в рамках госзадания Института оптики атмосферы им. В.Е. Зуева СО РАН.

\section{Конфликт интересов}

Авторы заявляют, что у них нет конфликта интересов.

\section{Список литературы}

[1] Таланов В.И. // Изв. вузов. Радиофизика. 1964. Т. 7. № 5. C. 564.

[2] Korobkin V.V., Alcock A.J. // Phys. Rev. Lett. 1968. V. 21. N 20. P. 1433.

[3] Braun A., Korn G., Liu X., Du D., Squier J., Mourou G. // Opt. Lett. 1995. V. 20. N 1. P. 73.

[4] Boyd R.W., Lukishova S.G., Shen Y.R. Self-focusing: past and present. Springer, 2009. $605 \mathrm{p}$. 
[5] Чекалин С.В., Кандидов В.П. // УФН. 2013. Т. 183. № 2. C. 133.

[6] Fedotov A.B., Koroteev N.I., Loy M.M.T., Xiao X., Zheltikov A.M. // Opt. Commun. 1997. V. 133. N 1-6. P. 587.

[7] Nibbering E.T.J., Curley P.F., Grillon G., Prade B.S., Franco M.A., Salin F., Mysyrowicz A. // Opt. Lett. 1996. V. 21. N 1. P. 62.

[8] Kosareva O.G., Kandidov V.P., Brodeur A., Chien C.Y., Chin S.L. // Opt. Lett. 1997. V. 22. N 17. P. 1332

[9] Rairoux P., Schillinger H., Niedermeier S., Rodriguez M., Ronneberger F., Sauerbrey R., Stein B., Waite D., Wedekind C., Wille H., Wöste L. // Appl. Phys. B. 2000. V. 71. N 4. P. 573.

[10] Kartashov D.V., Kirsanov A.V., Kiselev A.M., Stepanov A.N., Bochkarev N.N., Ponomarev Yu.N., Tikhomirov B.A. // Opt. Exp. 2006. V. 14. N 17. P. 7552.

[11] Киселев А.М., Пономарев Ю.Н., Степанов А.Н., Тихомиров А.Б., Тихомиров Б.А. // Квант. электрон. 2011. Т. 41. № 11. C. 976 .

[12] Ionin A.A., Kudryashov S.I., Ponomarev Y.N., Seleznev L.V., Sinitsyn D.V., Tikhomirov B.A. // AIP Conf. Proc. Santa Fe (New Mexico). 2010. V. 1278. P. 354. https://doi.org/10.1063/v1278.frontmatter

[13] Shutov A.V., Ustinovskii N.N., Smetanin I.V., Mokrousova D.V., Goncharov S.A., Ryabchuk S.V., Sunchugasheva E.S., Seleznev L.V., Ionin A.A., Zvorykin V.D. // Appl. Phys. Lett. 2017. V. 111. P. 224104-1-5.

[14] Синица Л.Н. Методы спектроскопии высокого разрешения / Учебное пособие. Томск: Томский государственный университет, 2006. 364 с

[15] Gordon I.E., Rothman L.S., Hill C., Kochanov R.V., Tan Y., Bernath P.F., Birk M., Boudon V., Campargue A., Chance K.V., Drouin B.J., Flaud J.-M., Gamache R.R., Hodges J.T., Jacquemart D., Perevalov V.I., Perrin A., Shine K.P., Smith M.-A.H., Tennyson J., Toon G.C., Tran H., Tyuterev Vl.G., Barbe A., Császár A.G., Devi V.M., Furtenbacher T., Harrison J.J., Hartmann J.-M., Jolly A., Johnson T.J., Karman T., Kleiner I., Kyuberis A.A., Loos J., Lyulin O.M., Massie S.T., Mikhailenko S.N., MoazzenAhmadi N., Müller H.S.P., Naumenko O.V., Nikitin A.V., Polyansky O.L., Rey M., Rotger M., Sharpe S.W., Sung K., Starikova E., Tashkun S.A., Vander Auwera J., Wagner G., Wilzewski J., Wcisto P., Yu S., Zak E.J. // JQSRT. 2017. V. 203. P. 3-69.

[16] Фомин Б.А., Колокутин Г.Э. // Современные проблемы дистанционного зондирования Земли из космоса. 2019. T. 16. № 1. C. 17.

[17] Делоне Н.Б. Взаимодействие лазерного излучения с веществом. М.: Наука, 1989. 280 с.

[18] Жаров В.П., Летохов В.С. Лазерная оптико-акустическая спектроскопия. М.: Наука, 1984. 320 с.

[19] Райзер Ю.П. Физика газового разряда. М.: Наука, 1992. $536 \mathrm{c.}$

[20] Heritier J.-M. // Opt. Commun. 1983. V. 44. N 4. P. 267.

[21] Протасевич А.Е., Тихомиров Б.А. // Оптика атмосферы и океана. 2011. Т. 24. № 4. С. 328.

[22] Okabe H. Photochemistry of small molecules. WileyInterscience, 1978. 431 р.; Окабе X. Фотохимия малых молекул. М.: Мир, 1981. 504 с.

[25] Куряк А.Н., Тихомиров Б.А. // Квант. электрон. 2020. Т. 50. № 9. C. 876.
[26] Bogumil K., Orphal J., Homann T., Voigt S., Spietz P., Fleischmann O.C., Vogel A., Hartmann M., Bovensmann H., Frerick J., Burrows J.P. // J. Photochem. Photobiol. A: Chem. 2003. V. 157. I. 2-3. Р. 167.

[27] Пихтин А.Н. Оптическая и квантовая электроника. М.: Высшая школа, 2001. 573 с. 